\begin{tabular}{|c|l|}
\hline Title & Dependence of fragmentation behavior of colloidal aggregates on their fractal structure \\
\hline Author(s) & Harada, S.; Tanaka, R.; Nogami, H.; Sawada, M. \\
\hline Citation & $\begin{array}{l}\text { Journal of Colloid and Interface Science, 301(1), 123-129 } \\
\text { https://doi.org/10.1016/.jcis.2006.04.051 }\end{array}$ \\
\hline Issue Date & $2006-09$-01 \\
\hline Doc URL & http://hdl.handle.net/2115/14634 \\
\hline Type & article (author version) \\
\hline File Information & JCI2006-301-1.pdf \\
\hline
\end{tabular}

Instructions for use 


\title{
Dependence of Fragmentation Behavior of Colloidal Aggregates on their Fractal Structure
}

\author{
S. Harada ${ }^{\dagger}$, R. Tanaka ${ }^{\dagger}$, H. Nogami $\mathrm{i}^{\ddagger}$ and M. Sawada
}

$\dagger$ Div. of Field Engineering for Environment, Graduate School of Eng., Hokkaido Univ. N13-W8, Sapporo 060-8628, Japan

$\ddagger$ Murata Manufacturing Company, Ltd.,

10-1, Higashi Kotari 1-chome, Nagaokakyo, Kyoto 617-8555, Japan

Corresponding Author : Shusaku Harada

Address : Division of Field Engineering for Environment,

Graduate School of Engineering, Hokkaido University

N13-W8, Sapporo 060-8628, Japan

$\mathrm{Tel} / \mathrm{Fax}:+81-11-706-6310$

E-mail : harada@eng.hokudai.ac.jp 


\begin{abstract}
The fragmentation dynamics of aggregate of non-Brownian particles in shear flow is investigated numerically. The breakup behaviors of aggregates having the same connectivity but the different space-filling properties are examined. The Lagrangian particle simulation in a linear flow field is performed. The effect of surrounding fluid on the motion of multiple particles is estimated by Stokesian dynamics approach. The inter-particle force is calculated from the retarded van der Waals potential based on the Lifshitz theory. The results obtained in this work indicate that the fragmentation behavior of colloidal aggregates depends on their fractal structure. However, if the resultant aggregate size is smaller than the critical one, the fragmentation behavior shows the universality regardless of their original structure. Furthermore, the restructuring of aggregate in shear flow and its effect on the fragmentation process are also discussed.
\end{abstract}

Keywords : aggregate; breakup; shear flow; numerical analysis; restructuring; Stokesian dynamics 


\section{Introduction}

The aggregation and the dispersion processes of colloidal particles are widely seen in applications related to chemical, electrical and environmental engineering. Not only do they influence the rheological properties of colloidal suspensions, but they play important roles in handlings of solid particle such as the liquid-solid separation, the coating of particles on a surface and the formation of the crystalline assemblies. The prediction and the control of aggregate size in fluid having various static and dynamic properties are required for a lot of processes in which nano- and micro-particles are treated.

It is known that the colloidal aggregate has a wide variety of structures even if it grows in a static fluid, and the structure depends on the dominant force acting on particles during the growth[1]. For instance, if particles are adequately small and Brownian effect dominates the particle motion, the aggregate has many branches and is called Brownian aggregate or diffusion-limited aggregate (DLA). On the other hand, if a potential barrier between particles exists, the aggregate is more compact than DLA and it is called reaction-limited aggregate (RLA). Therefore the structure of aggregate is determined by physical and chemical properties of suspension and thermodynamic conditions during the growth.

The variety of the aggregate structure prevents us from predicting the dispersion state in fluid flow quantitatively. In a dynamic system of colloidal dispersion, where both the fluid and the suspended particles are in motion, the aggregation and/or the dispersion occur due to hydrodynamic force[2]. The former is called flow-induced aggregation and is the process that the particle or the cluster which moves in flow field meets other ones and they become united. It is obvious that the structure of aggregate influences their "collision" frequency. Thus the aggregation process is dependent on their structures. The latter means that the hydrodynamic stress breaks up the aggregate particles. This fragmentation process has been studied by a lot of experimental and theoretical approaches[3]-[7]. In most of the theoretical studies, an aggregate is simplified as a solid having internal cohesive strength, and the breakup occurs due to the hydrodynamic stress in excess of the cohesive strength (critical stress). If the stress larger than the critical one is applied, the aggregate size gets smaller with increasing hydrodynamic stress. In a simple shear flow, some researchers pointed out the relation between hydrodynamic shear stress characterized by $\mu \dot{\gamma}(\mu$ : viscosity, $\dot{\gamma}$ : shear rate) and the mean aggregate size obeys the power-law $[7,8]$. The exponent of the power-law is called fracture exponent and it depends mainly on the fractal dimension of aggregates.

Accordingly, the stable size of aggregate for a given flow condition is largely dependent on the aggregate structure. If the prediction and the control of the aggregate size in a shear flow is required, the following information is needed; 1) the original size and the internal structure of the resultant aggregates for a given condition, 2) the critical stress for fragmentation and 3) the fracture exponent of the aggregate (and also the limit of power-law dependence of aggregate size on shear stress). Moreover, it is possible to occur 4) the restructuring of aggregate in flow field[9] and 5) the re-aggregation of clusters during the fragmentation process[10, 11].

The purpose of this study is the fundamental understanding of the above 2) to 4) effects, that is, the fragmentation of an isolated aggregate with an arbitrary internal structure in a simple shear flow. We investigated those effects by the numerical analysis. There have been some numerical studies on the dynamics of an aggregate in a flow field by using fluid force models[12]-[14]. Most 
of the numerical studies (and also the theoretical analyses by fragmentation model) considered the hydrodynamic force acting on constitutive particles as the surface force on the aggregate. However, the hydrodynamic interaction between primary particles is important for the fragmentation of aggregate. The short-range hydrodynamic interaction (lubrication effect) directly concerns the separation of primary particles while the long-range interaction decides the relative motion of ruptured aggregates. For the quantitative understanding of the fragmentation process, the accurate estimation of these hydrodynamic interactions of particles are required. Thus we adopted the Stokesian dynamics approach for the calculation of hydrodynamic force acting on particles in order to calculate the wide range of the hydrodynamic interaction accurately. In this article, we focus on a dynamic behavior of aggregates before and after breakup, and discuss the influence of the structure of aggregates to the critical stress.

\section{Numerical Methods}

The motion of colloidal particles in flow field is simulated by Stokesian dynamics approach. On the assumption that the particle is adequately small, the inertias of both particle and fluid are neglected. The mobility matrix of particle is derived from multipole expansion of Oseen tensor in Stokes flow and Faxén law. By the mobility matrix, the external force, torque and the stresslet are related to the particle velocity, angular velocity and the rate of strain tensor of the undisturbed flow field. The detailed procedure of the calculation is the same as Durlofsky et al. [15] and the full description of the mobility matrix can be found in their articles. The outline of the calculation procedure is shown below.

The grand mobility matrix which describes the mobility of all particles $M$ is inverted, and $M^{-1}$ is divided in the following components.

$$
\begin{gathered}
\left(\begin{array}{c}
\boldsymbol{F} \\
\boldsymbol{T} \\
\boldsymbol{S}
\end{array}\right)=\boldsymbol{M}^{-\mathbf{1}}\left(\begin{array}{c}
\boldsymbol{U}-\boldsymbol{u}^{\infty} \\
\boldsymbol{\Omega}-\boldsymbol{\Omega}^{\infty} \\
-\boldsymbol{E}^{\infty}
\end{array}\right), \\
\boldsymbol{M}^{-\mathbf{1}}=\left(\begin{array}{lll}
\boldsymbol{R}_{F U} & \boldsymbol{R}_{F \Omega} & \boldsymbol{R}_{F E} \\
\boldsymbol{R}_{T U} & \boldsymbol{R}_{T \Omega} & \boldsymbol{R}_{T E} \\
\boldsymbol{R}_{S U} & \boldsymbol{R}_{S \Omega} & \boldsymbol{R}_{S E}
\end{array}\right),
\end{gathered}
$$

where $\boldsymbol{F}, \boldsymbol{T}$ and $\boldsymbol{S}$ are the external force, torque acting on all particles and stresslet, $\boldsymbol{U}$ and $\boldsymbol{\Omega}$ are the particle velocity and the rotational velocity, $\boldsymbol{u}^{\infty}, \boldsymbol{\Omega}^{\infty}$ and $\boldsymbol{E}^{\infty}$ are the flow velocity, rotational velocity and the rate of strain tensor respectively. Based on the notation of Eq.(2), the velocity and the angular velocity of individual particles are calculated as

$$
\left(\begin{array}{c}
\boldsymbol{U} \\
\boldsymbol{\Omega}
\end{array}\right)=\left(\begin{array}{c}
\boldsymbol{u}^{\infty} \\
\boldsymbol{\Omega}^{\infty}
\end{array}\right)+\left(\begin{array}{cc}
\overline{\boldsymbol{R}}_{F U} & \overline{\boldsymbol{R}}_{F \Omega} \\
\overline{\boldsymbol{R}}_{T U} & \overline{\boldsymbol{R}}_{T \Omega}
\end{array}\right)^{-\mathbf{1}}\left[\left(\begin{array}{c}
\boldsymbol{F} \\
\boldsymbol{T}
\end{array}\right)+\left(\begin{array}{c}
\overline{\boldsymbol{R}}_{F E} \\
\overline{\boldsymbol{R}}_{T E}
\end{array}\right): \boldsymbol{E}^{\infty}\right]
$$

where $\overline{\boldsymbol{R}}$ is the modified resistance matrix by lubrication correlation and is obtained as follows;

$$
\overline{\boldsymbol{R}}=\boldsymbol{R}+\boldsymbol{R}_{2 B}-\left(M_{2 B}\right)^{-\mathbf{1}},
$$

where $\boldsymbol{R}_{2 B}$ is the resistance matrix for a pair of particles[18, 19], $\left(\boldsymbol{M}_{2 B}\right)^{-1}$ is the inverse of the pair mobility matrix calculated by the same way as $M^{-1}$. The instantaneous position of particles is calculated by numerical integration of Eq.(3). 
The particles are assumed to be perfectly smooth spheres with the diameter $2 a=650 \mathrm{~nm}$. The Brownian perturbation force is neglected on the assumption of large Péclet number $P e=$ $6 \pi \mu a^{3} \dot{\gamma} / k T$ as described below. The retarded van der Waals force is given as an inter-particle force and it is calculated by the rigorous approach which is the combination of retarded Hamaker constant based on Lihshitz theory and a Hamaker geometrical factor[16, 17]. For the physical properties of particle and fluid, those of polystyrene and ethanol are used. The particle and the fluid density are $\rho_{p}=1056 \mathrm{~kg} / \mathrm{m}^{3}, \rho_{f}=790 \mathrm{~kg} / \mathrm{m}^{3}$ respectively and the fluid viscosity is $\mu=1.2 \times 10^{-3} \mathrm{~Pa} \cdot \mathrm{s}$. If the distance between particles is closer than the contact distance $\delta=1 \mathrm{~nm}$, the inter-particle van der Waals force acting on them is set equal to zero and any repulsive force is not given them. Owing to lubrication force, any overlap of particles (i.e., the distance between the centers of the particle $r<2 a$ ) does not occur in this simulation. The maximum attractive inter-particle force, which is important for the estimation of cohesive strength of particles on the breakup process, is approximately $F_{M}=A a / 12 \delta^{2}$ where $A$ is non-retarded Hamaker constant and $A=9.68 \times 10^{-21} \mathrm{~J}$ in this condition. As a result, the force needed to separate a couple of particles $F_{M}$ is $2.62 \times 10^{-10} \mathrm{~N}$.

The simulation of the motion of an isolated aggregate in simple shear flow is performed by the method explained above. The schematic diagram and the coordinate system of the calculation is shown in Fig.1. The surrounding flow field is expressed as

$$
u^{\infty}(r)=\Omega^{\infty} \times r+E^{\infty} \cdot r
$$

where

$$
\Omega^{\infty}=-\frac{\dot{\gamma}}{2}\left(\begin{array}{l}
0 \\
0 \\
1
\end{array}\right), \quad \boldsymbol{E}^{\infty}=\frac{\dot{\gamma}}{2}\left(\begin{array}{lll}
0 & 1 & 0 \\
1 & 0 & 0 \\
0 & 0 & 0
\end{array}\right) .
$$

In order to investigate the effect of the internal structure of the aggregate on the fragmentation, we prepare two kinds of aggregates (called parent aggregates) which consist of a hundred primary particles as the initial condition. Their appearances are shown in Fig.2. Figure 2(a) shows the aggregate called DLCA (diffusion-limited cluster aggregate) and it is formed by the collision of DLA clusters in the same way as Sutherland et al. [20]. Figure 2(b) is called LTA (linear trajectory aggregate) and it is made by the one-point contact between a cluster of particles and a particle which distributes in space at random and moves with a linear trajectory toward the cluster. Their fractal dimensions are $F r=1.9$ (DLCA) and $F r=3.0$ (LTA) respectively. The average coordination number, which is defined by the average number of particles existing within the distance $2 \delta$ from a particle, is $k=2.0$ for both DLCA and LTA. While the fractal dimension characterizes the space-filling properties of an aggregate, the coordination number does the connectivity of primary particles. By the comparison between DLCA and LTA, we could examine the fragmentation behavior of aggregates having the same connectivity but the different space-filling properties.

The verification of Stokesian dynamics simulation is performed elsewhere[21]. Here we demonstrate the sedimentation of multi-particles in static fluid. Figure 3 shows the numerical results of the settling motion of three unequally spaced spheres. The results in the same conditions obtained from the boundary collocation method by Ganatos et al.[22] are also shown in the figure. It is found that unequally spaced spheres settle with the change in their relative positions. Figure 3 shows that our results agree with the results by Ganatos et al. quantitatively in terms of the settling distance, the velocity and the relative position of particles. These agreements imply that 
both the short- and the long range hydrodynamic interaction between particles could be calculated properly by our simulation method.

Final part of this section, we discuss the applicability of the assumptions used in this study. The first assumption is the neglect of both the particle and fluid inertia, which is correspondent to the dimensionless parameters $R e=\rho_{f} a U / \mu$ and $S t=\rho_{p} a U / \mu$ less than unity. The particle and fluid properties shown above restrict the characteristic velocity $U$ to less than $O\left(10^{0}\right) \mathrm{m} / \mathrm{s}$. Two kinds of the characteristic velocity of the system can be defined. One is derived from a balance of the inter-particle force characterized by $A / a$ and the drag force $6 \pi \mu a U$, and then $U=A / 6 \pi \mu a^{2}$. The other is based on the shear rate and the particle radius $a \dot{\gamma}$. The former expresses the characteristic velocity of the response of the aggregation and is sufficiently less than unity in this condition $\left(U<O\left(10^{-5}\right) \mathrm{m} / \mathrm{s}\right)$. The latter is the characteristic velocity of surrounding shear flow, and the condition $\mu \dot{\gamma}<O\left(10^{4}\right)$ Pa must be satisfied for $R e, S t<1$. In this study, the calculation condition of the shear rate is set $\mu \dot{\gamma}<200 \mathrm{~Pa}$, hence the above restrictions of the characteristic velocity are satisfied.

The second assumption concerns Brownian motion. In shear flow, the neglecting of Brownian motion is the reasonable assumption if the Péclet number $P e=6 \pi \mu a^{3} \dot{\gamma} / k T$ is sufficiently large. In this calculation, $\mathrm{Pe}>O\left(10^{4}\right)$ is satisfied for all conditions. The microscopic effect of the Brownian perturbation to the strength of individual bond between particles is still not clear, but we neglect it.

The last is the applicability of the calculation conditions. As mentioned above, we set the contact distance $\delta=1 \mathrm{~nm}$ and set the inter-particle force zero if particles approach within $\delta$. This condition affects the dispersion behavior of particles because the inter-particle attractive force is proportional to $\delta^{-2}$. In general, the length-scale of the contact distance is considered several angstroms [23, 24]. However, various complex effects caused by the molecular nature of the sorrounding fluid i.e., the structural force, the lubrication effect at molecular scale and also the Brownian effect, are not negligible in such a small length-scale. In addition, the surface of the primary particle in actual system is not smooth and is possible to have an angstromorder roughness. It is difficult to estimate these molecular effects rigorously and to include in the analysis of particle dynamics. Therefore we ignored these microscopic effects and angstrom-order interparticle force. This assumption may cause some quantitative difference between the dispersion behavior of the actual system with substances given above and that obtained from the numerical analysis. However, we verified that the discussion about the effect of the aggregate structure on the fragmentation process given in this article is not influenced by the estimation of the contact distance.

The calculation condition of the time step also influences the fragmentation process of particles. Owing to discretization, the relative motion between particles is digitalized and consequently the force needed to separate a couple of particles is underestimated. We decided the time step by preliminary calculations of the motion of a couple of particles which exert an inter-particle force. We set the time step so that the numerical error of the force required to the breakup is almost the same in all conditions and is within $8 \%$ of the exact value of $F_{M}$.

\section{Results and discussion}

Figures 4 and 5 show the numerical results of the motion of DLCA and LTA in shear flow. In these figures, the behaviors of aggregate are shown at regular time intervals from their initial 
states although both figures have a different time interval. The flow condition is common to these calculations and the aggregates are exposed to shear flow with $\mu \dot{\gamma}=44.9 \mathrm{~Pa}$. It is found that both aggregates are ruptured and divide into small aggregates with several or several tens of particles. The fragmentation process of DLCA at early stage can be explained by division of branches and the aggregate size is almost decided at this stage. Then isolated branches are restructured and some of them are rounded with the rotation of flow individually. As a result, each small aggregate has lost the fractal appearance their parent aggregate had. On the other hand, the breakup of LTA shows a different behavior from that of DLCA. The parent aggregate keeps rotation with the rotation of surrounding flow and deforms gradually with rotation. After a while, it ruptures and divide into small aggregates. In other words, LTA changes the appearance before breakup, while DLCA changes it after breakup.

Figure 6 shows the relation between the hydrodynamic stress $\mu \dot{\gamma}$ and the weight average number of primary particles which compose ruptured aggregate. As shown in Fig.6, the average number of particles decreases with the increase in shear rate and is a power-law dependence of $\mu \dot{\gamma}$. However, the fragmentation trend of low shear region is different from that of high shear region. If the high shear rate is applied, the relation between $\mu \dot{\gamma}$ and the average number of particles is similar in case of these aggregates. In low-shear region, the fragmentation of both aggregates shows different behavior. For DLCA, the fracture exponent (the exponent of power-law) in the low-shear region is larger than that in the high-shear region and it is almost the same as the experimental results for the fractal aggregate $(F r=2.2)$ by Sonntag and Russel[8]. In case of LTA, the size of ruptured aggregate in low shear is scattering. It is also found that the critical shear stress, which is the shear stress in excess of that requires their breakup, of LTA is almost ten times as large as that of DLCA.

The agreement of the fracture exponent of these aggregates for high shear rate is explained by the disappearance of the original structure of the parent aggregate with the decrease in the size of child aggregate. This implies that the breakup behavior below the critical size is independent of the fractal structure that the parent aggregate had. The critical number of primary particles can be estimated from Fig. 6 and is about ten. The quantitative meaning of this critical size is under consideration.

Figure 7 is the relation between the fractal dimension of aggregate and the fracture exponent given by previous studies. Horwatt et al.[7] analyzed the hydrodynamic dispersion of an aggregate having heterogeneous structures by the fragmentation model. In their model, the balance between the hydrodynamic force acting on the surface of an isolated spherical aggregate and the cohesive force due to the heterogeneous interparticle bond in it is considered. They obtained the fracture exponent of aggregate having various structures and showed that the aggregate with $F r<2.4$, the fracture exponent is almost constant and is about -0.8 . However, the exponent of the aggregate with $F r>2.4$ decreases with increasing $F r$ and it is difficult to characterize their fragmentation behavior in terms of only their fractal dimension (See the hatched region in Fig.7). Some experimental measurements of the size of ruptured aggregate support their results[6, 8]. In Fig.7, our simulation results are also plotted. The fracture exponent for DLCA in lower shear rate is plotted at $F r=1.9$ and the exponent for DLCA and LTA in higher shear rate is plotted at $F r=3.0$. Their values are already shown in Fig.6 respectively. For lower $F r$, our result quantitatively agrees with 
previous results, and is not inconsistent with the results by the fragmentation model for higher Fr.

The results shown above indicate that the structure of parent aggregate affects the size of child aggregates and that the aggregate having higher fractal dimension is more difficult to breakup by fluid shear stress. The relation between the shear rate and the size of ruptured aggregate obeys the power-law with their respective exponents if the aggregate size is larger than the critical size. In case of the smaller aggregates, the fracture exponent is independent of the structure and the breakup behavior can be characterized in terms of only applied shear stress.

We assume that the scatter of breakup behavior of LTA for low shear rate is caused by the change of their structure in flow field. In case of the fractal aggregate, it is known that the restructuring occurs with the motion in flow field. That is, the deformation and the formation of new bonds between neighboring particles occur due to the relative motion of constituent particles.

In order to examine the dynamics of restructuring of non-fractal aggregate and its effect on the fragmentation process, the change in the structure of LTA before breakup was examined. Figure 8 shows the typical examples of the configurations of LTA in shear flow for $\mu \dot{\gamma}=26.9$ and $44.9 \mathrm{~Pa}$ before breakup. It is found that the parent aggregate deforms with the rotation and the particle forms new bonds to neighboring particles, i.e., the restructuring occurs. As a result, the packing state changes from loose to dense ones as shown later.

Figure 8 also shows that the configuration of the aggregate changes considerably before breakup with the development of the deformation. The aggregate is stretched along the streamlines and then it bends with rotation. Such a deformation process of non-fractal aggregate is very similar to the experimental observation by Blaser (See Fig.4 in Ref.[25]).

Figure 9 shows the change in the average coordination number of LTA at the early stage. Some conditions of the shear rate of the flow are correspondent to those shown in Fig.8. In Fig.9, the line branches off indicates that the aggregate ruptures and separates into some child aggregates. We considered that the ruptured aggregates with the number of the constituent particles less than ten are the "erosion" aggregate and their coordination number is not shown in the figure. As shown in Fig.9, the coordination number increases with the non-dimensional time $\dot{\gamma} t$ which is the time normalized by the characteristic time of the rotation (and also the deformation) of the flow field $1 / \dot{\gamma}$. It is found that the restructuring process is well scaled by $\dot{\gamma} t$. For $\mu \dot{\gamma}=26.9$ Pa, the coordination number is changed from the initial value $k=2.0$ to 5.5 by $\dot{\gamma} t=80$. This is explained that the restructuring makes the aggregate denser and consequently the coordination number reaches the maximum number. The coordination number of closest random packing of spherical particle can found elsewhere and the coordination number $k=5.5$ is somewhat less than the well-known value ( $k=6.0 \sim 8.0$ for closest random packing). This is because the aggregate considered in our simulation is so small that the number of particles near the aggregate surface, which cannot bond the neighboring particles sufficiently, is comparable with the total number of particles.

As also shown in Fig.9, the breakup is observed on the way of the restructuring for $\mu \dot{\gamma}=38.8$ and $44.9 \mathrm{~Pa}$. These results suggest that the size of child aggregate of LTA depends not on the initial structure but on the instantaneous structure just before breakup. That is, the resultant aggregate size is related to the progression of the restructuring before breakup. 
The restructuring of LTA explains the scattering of fragmentation process of LTA shown in Fig.6. DLCA has a lot of branches because of its fractal structure and the breakup easily occurs at branch point due to the strain effect of shear flow. In case of LTA, which has a loose and non-fractal structure, the restructuring easily occurs in shear flow and the parent aggregate ruptures on the way of the deformation. Therefore the aggregate size is not determined definitely by the shear rate. The upper boundary of the hatched region in Fig.6, which indicates the maximum aggregate size of LTA for a given shear rate, shows the similar fracture exponent as that in high-shear region. This suggests that there is the limit of the effect of the restructuring because the fragmentation process in high-shear region is independent of the aggregate structure as explained above. On the other hand, the minimum aggregate size of LTA (i.e., the lower boundary of the hatched region) is always larger than that of DLCA, although the coordination number of parent aggregate of LTA is the same as that of DLCA. This indicates that the fractal aggregate is more fragile than the non-fractal aggregate having the same cohesive strength in the average sense, even if the restructuring effect does not appear. This is consistent with the results derived from the heterogeneous fragmentation model by Horwatt et al.. (See Fig.7. The fracture exponent for $F r=3.0$ is always smaller than that for less $F r$.)

\section{Conclusions}

Numerical studies on the dynamics of colloidal aggregates in shear flow have been performed. The relationship between the hydrodynamic stress and the size of the ruptured aggregate has been examined. It is found that the breakup behavior of aggregates is largely dependent on their fractal structures. Moreover, the restructuring of aggregate plays an impotant role on the rupture process of non-fractal aggregates. We conclude that the fragmentation of aggregates in shear flow is influenced by both the strain effect and the rotation effect of shear flow. 


\section{References}

[1] P. Meakin, Adv. Colloid Interface Sci. 28 (1988) 249.

[2] J.L. Ottino, P. DeRoussel, S. Hansen, D.V. Khakhar, Adv. Chem. Eng. 25 (2000) 105.

[3] D.F. Bagster, D. Tomi, Chem. Eng. Sci. 29 (1974) 1773.

[4] P.M. Adler, P.M. Mills, J. Rheology 23 (1979) 25.

[5] R.C. Sonntag, W.B. Russel, J. Colloid Interface Sci. 115 (1987) 378.

[6] S.P. Rwei, I. Manas-Zloczower, D.L. Feke, Polymer Eng. Sci. 30 (1990) 701.

[7] S.W. Horwatt, I. Manas-Zloczower, D.L. Feke, Chem. Eng. Sci. 47 (1992) 1849.

[8] R.C. Sonntag, W.B. Russel, J. Colloid Interface Sci. 113 (1986) 399.

[9] M.Y. Lin, R. Klein, H.M. Lindsay, D.A. Weitz, R.C. Ball, P. Meakin, J. Colloid Interface Sci. 137 (1990) 263.

[10] V. Oles, J. Colloid Interface Sci. 154 (1992) 351.

[11] T. Serra, X. Casamitjana, J. Colloid Interface Sci. 206 (1998) 505.

[12] M. Doi, D. Chen, J. Chem. Phys. 90 (1989) 5271.

[13] K. Higashitani, K. Iimura, H. Sanda, Chem. Eng. Sci. 56 (2001) 2927.

[14] M. Fanelli, D.L. Feke, I. Manas-Zloczower, Chem. Eng. Sci. 61 (2006) 473.

[15] L. Durlofsky, J.F. Brady, G. Bossis, J. Fluid Mech. 180 (1987) 21.

[16] H.C. Hamaker, Physica 4 (1937) 1058.

[17] W.R. Bowen, F. Jenner, Adv. Colloid Int. Sci. 56 (1995) 201.

[18] S. Kim, S. J. Karrila, Microhydrodynamics, Butterworth-Heinemann, 1991.

[19] D.J. Jeffery, Y. Onishi, J. Fluid Mech. 139 (1984) 261.

[20] D.N. Sutherland, J. Colloid Interface Sci. 25 (1967) 373.

[21] J.F. Brady, G. Bossis, Ann. Rev. Fluid Mech. 20 (1988) 111.

[22] P. Ganatos, R. Pfeffer, S. Weinbaum, J. Fluid Mech. 84 (1978) 79.

[23] J.N. Israelachvili, Intermolecular and Surface Forces, Academic Press, 1992.

[24] N. Yu, A.A. Polycarpou, J. Colloid Int. Sci. 278 (2004) 428.

[25] S. Blaser, J. Colloid Interface Sci. 225 (2000) 273. 
Fig.1 Schematic diagram of calculation system

Fig.2 Structure of parent aggregates consisting 100 particles

Fig. 3 Instantaneous motion of three unequally spaced spheres. $\tilde{t}:$ dimensionless time $\left(=U_{t} t / a, U_{t}\right.$ : terminal velocity), $\tilde{d}$ : dimensionless settling distance $(=d / a, d$ : settling distance $), C$ : ratio of initial distance between spheres 1 and 2 to 2 and 3 . The initial distance between 1 and 3 is 12 radii

Fig. 4 Instantaneous motion of the fragmentation of DLCA aggregate for $\mu \dot{\gamma}=44.9 \mathrm{~Pa}$ in steps of dimensionless time $\dot{\gamma} t=1.40$

Fig. 5 Instantaneous motion of the fragmentation of LTA aggregate for $\mu \dot{\gamma}=44.9 \mathrm{~Pa}$ in steps of dimensionless time $\dot{\gamma} t=4.90$

Fig.6 Relation between $\mu \dot{\gamma}$ and weight average number of particles for DLCA and LTA with experimental results for fractal aggregate $(F r=2.2)[8]$

Fig.7 Fracture exponent by numerical simulations and experiments

Fig. 8 Configurations of LTA during restructuring in shear flow

Fig.9 Average coordination number of LTA during restructuring 


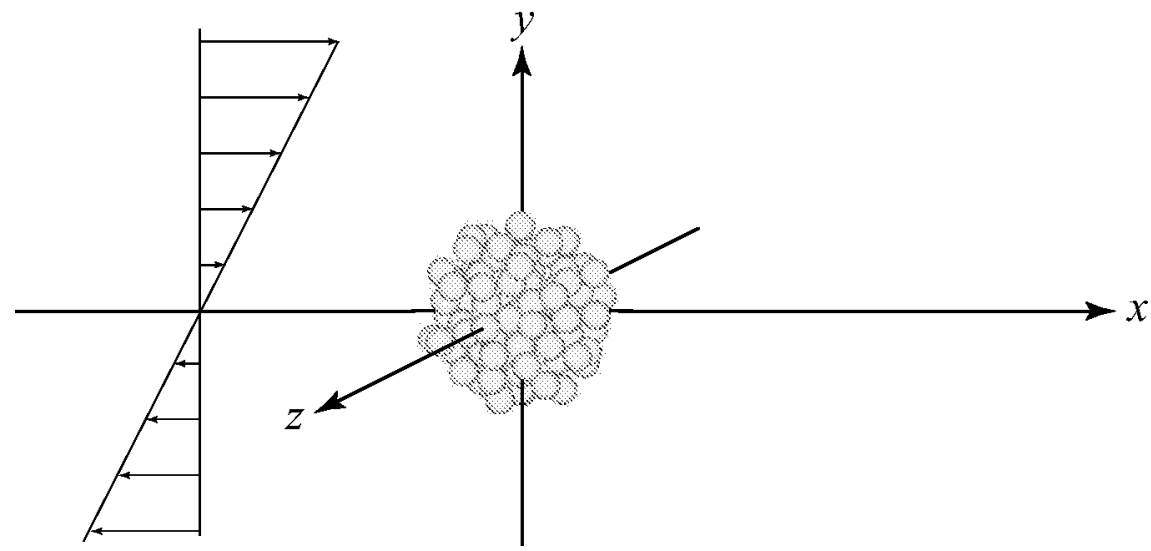

Fig.1 Schematic diagram of calculation system 


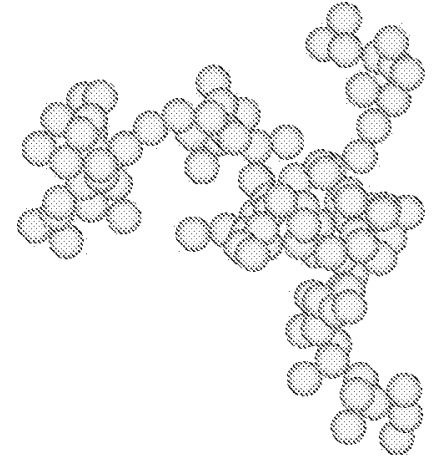

(a) DLCA

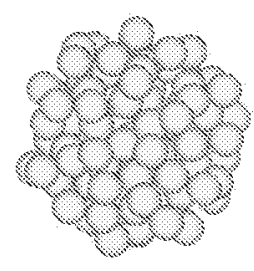

(b) LTA

Fig.2 Structure of parent aggregates consisting 100 particles 


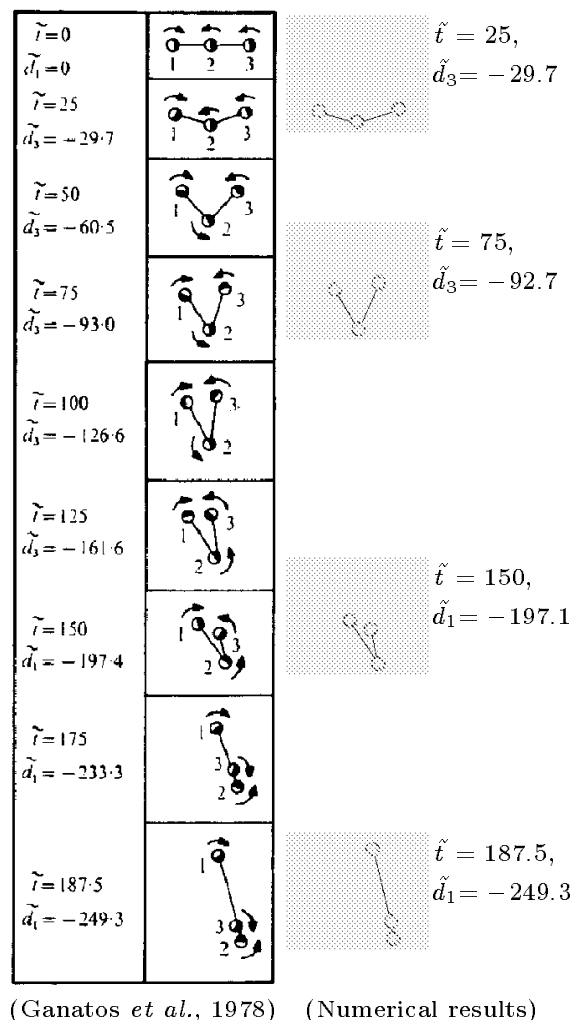

(a) $C=1.1$

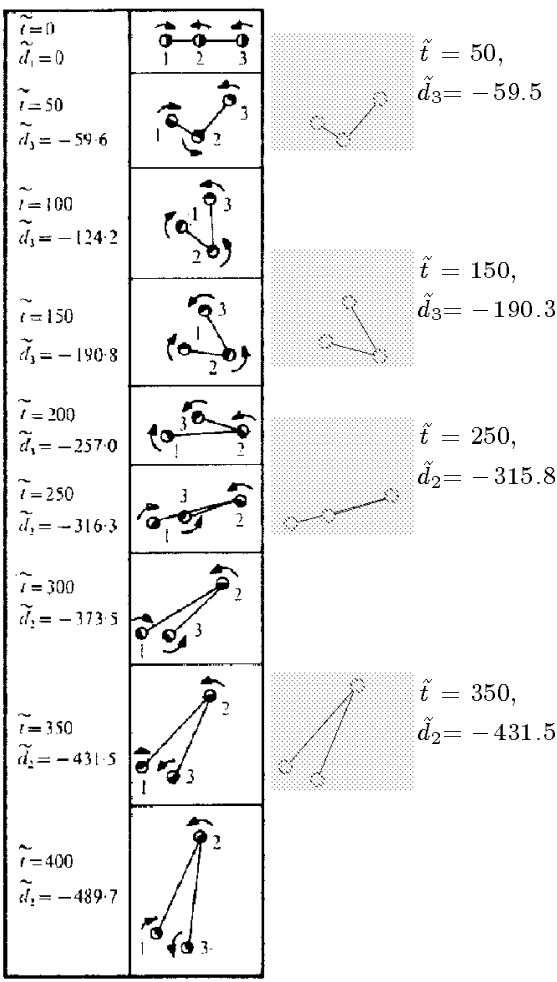

(Ganatos et al., 1978) (Numerical results)

(b) $C=1.6$

Fig.3 Instantaneous motion of three unequally spaced spheres. $\tilde{t}$ : dimensionless time (= $U_{t} t / a, U_{t}$ : terminal velocity), $\tilde{d}$ : dimensionless settling distance $(=d / a, d$ : settling distance), $C$ : ratio of initial distance between spheres 1 and 2 to 2 and 3 . The initial distance between 1 and 3 is 12 radii 


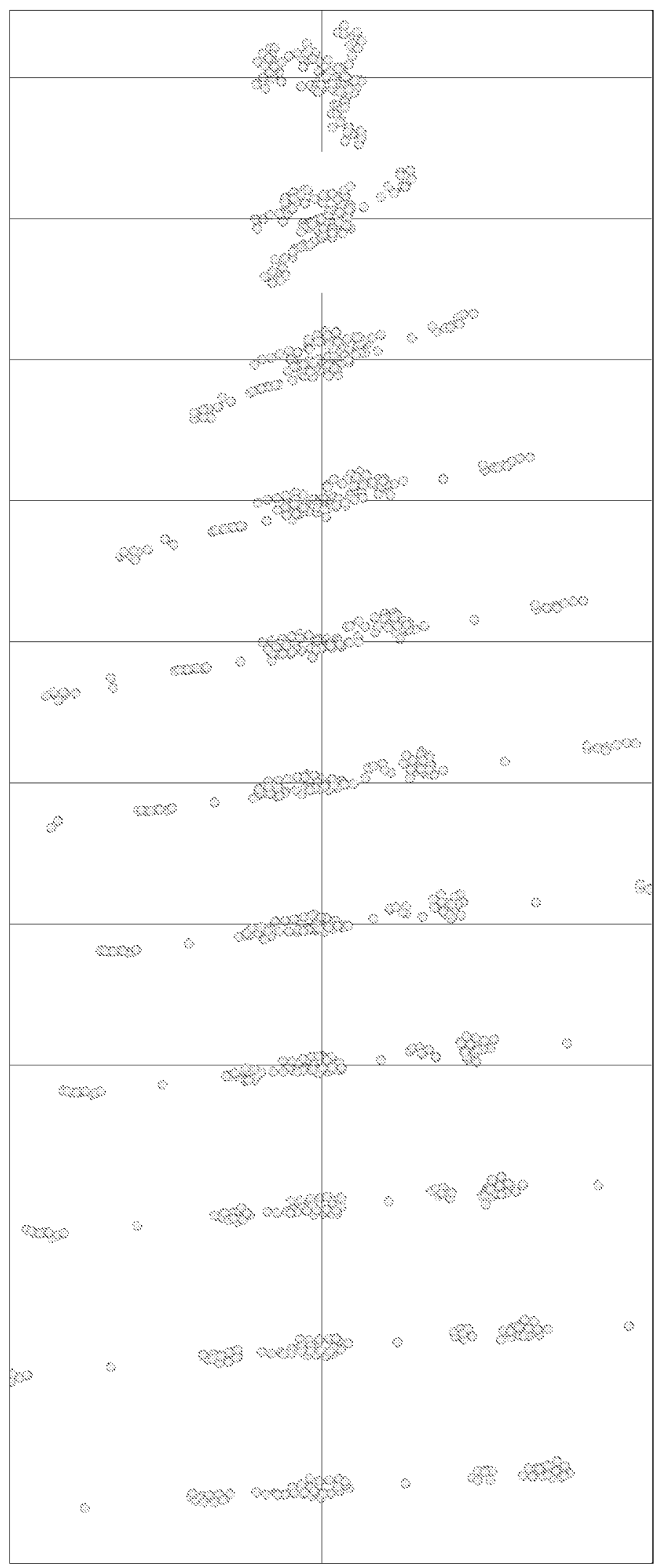

Fig.4 Instantaneous motion of the fragmentation of DLCA aggregate for $\mu \dot{\gamma}=44.9 \mathrm{~Pa}$ in steps of dimensionless time $\dot{\gamma} t=1.40$ 


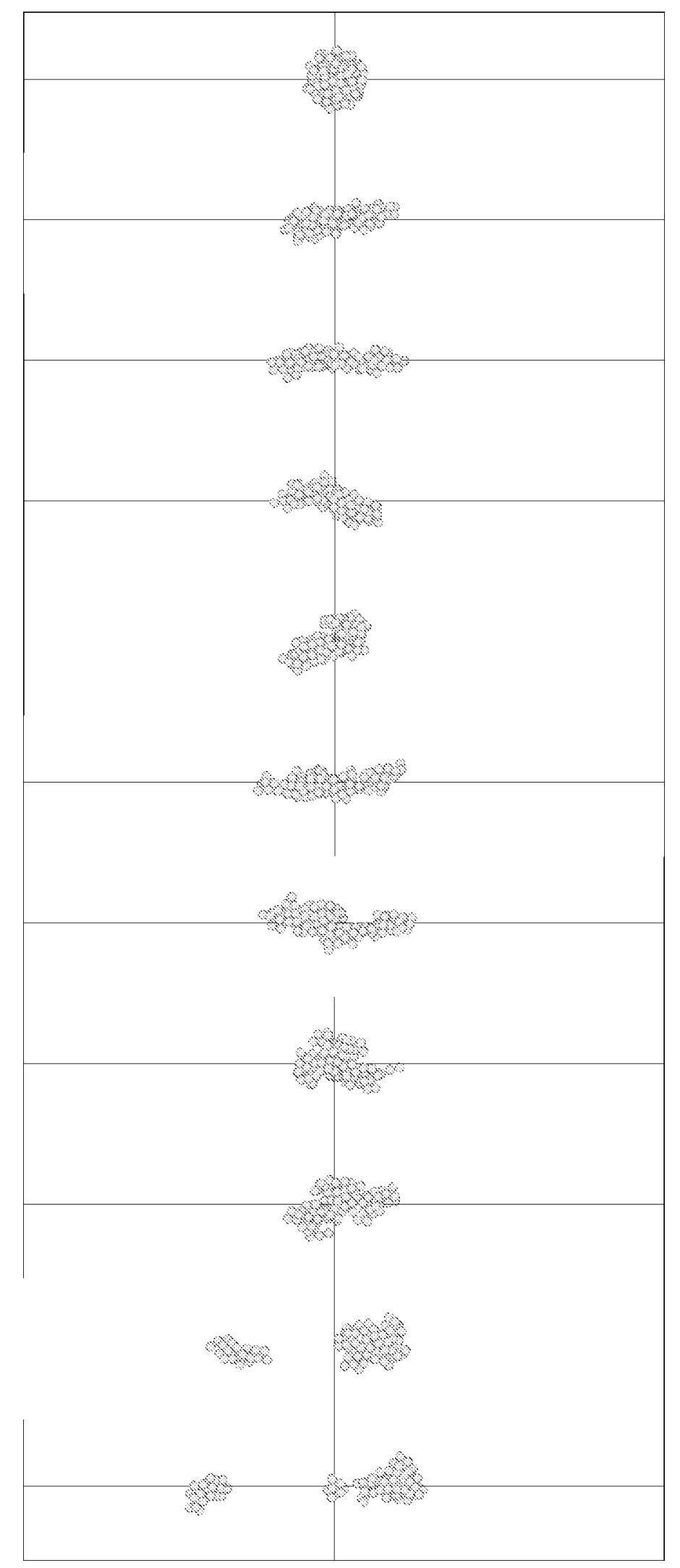

Fig.5 Instantaneous motion of the fragmentation of LTA aggregate for $\mu \dot{\gamma}=44.9 \mathrm{~Pa}$ in steps of dimensionless time $\dot{\gamma} t=4.90$ 


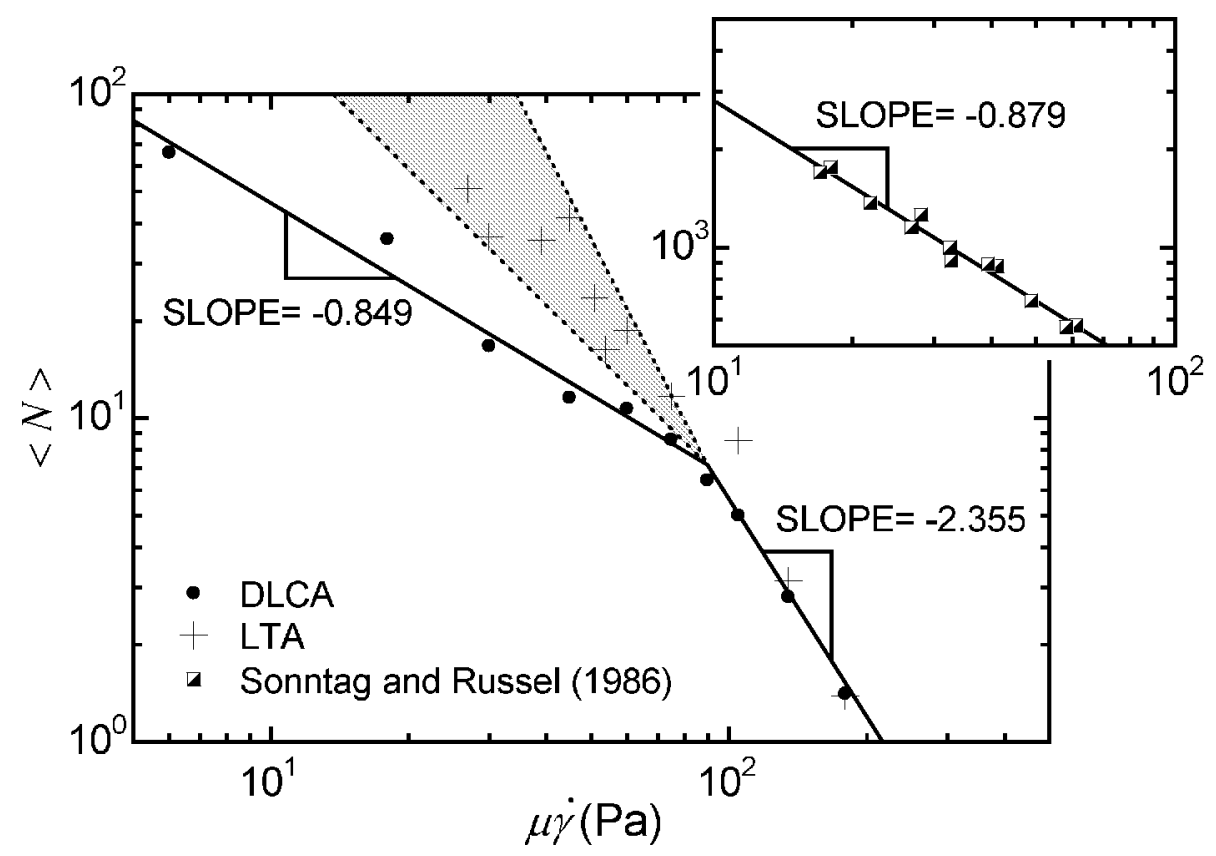

Fig.6 Relation between $\mu \dot{\gamma}$ and weight average number of particles for DLCA and LTA with experimental results for fractal aggregate $(F r=2.2)[8]$ 


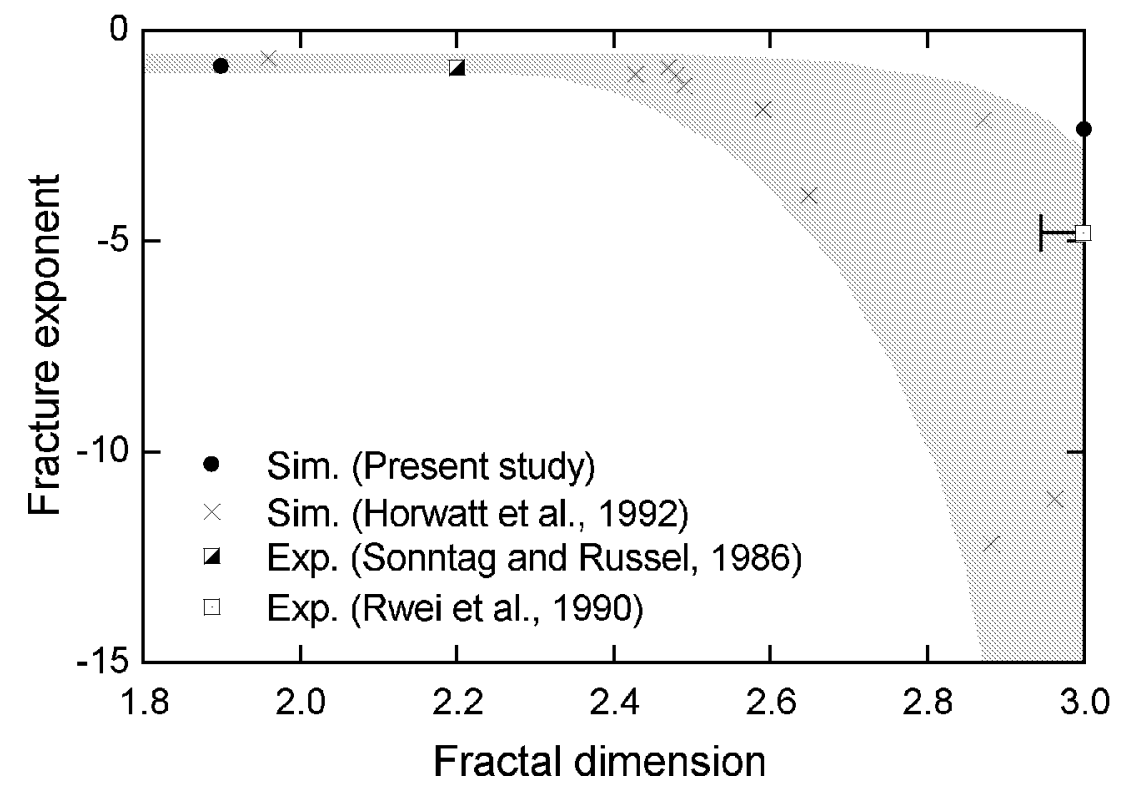

Fig.7 Fracture exponent by numerical simulations and experiments 


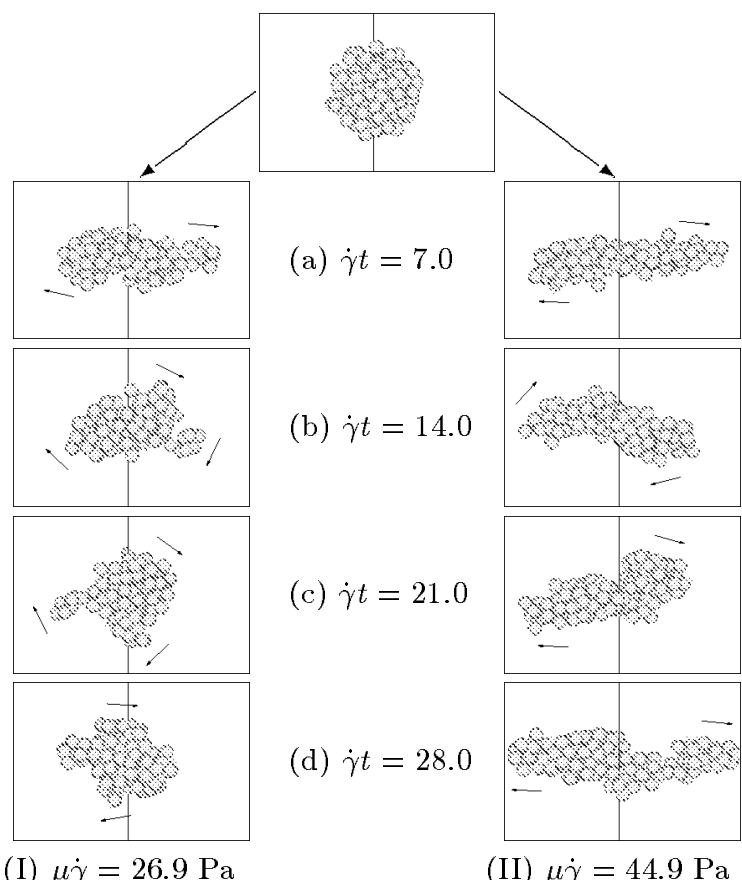

Fig.8 Configurations of LTA during restructuring in shear flow 


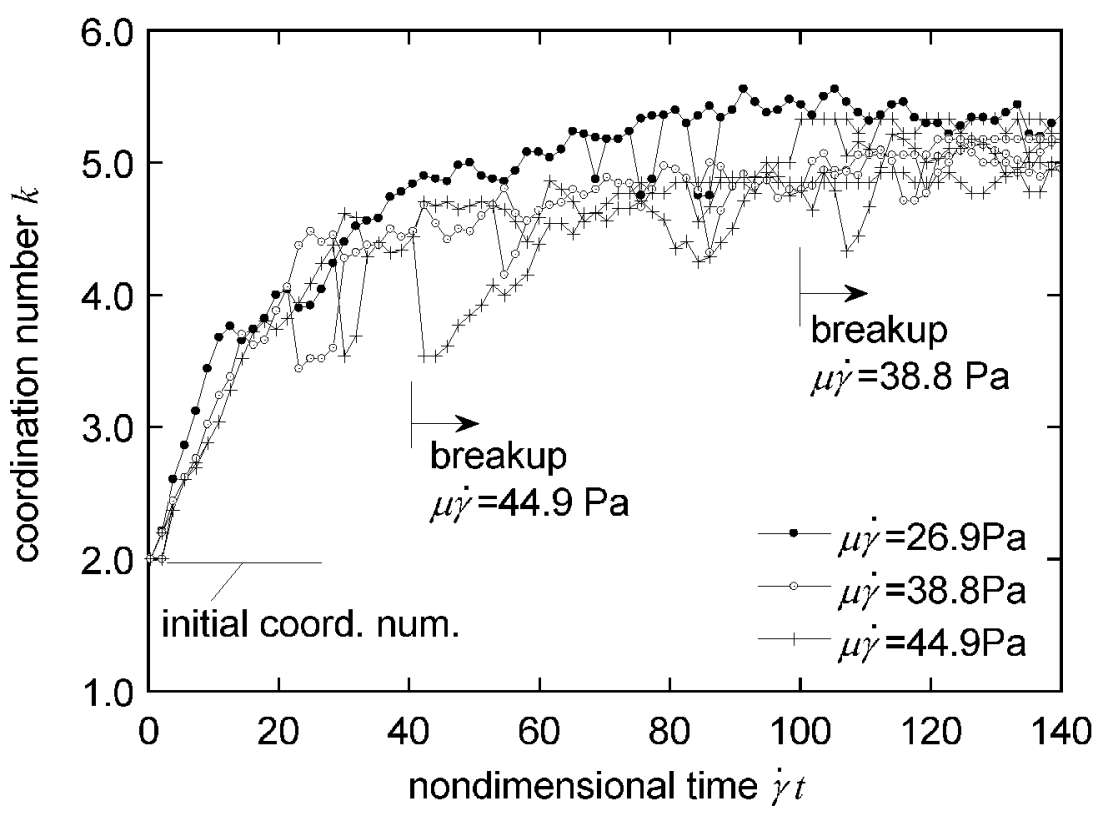

Fig.9 Average coordination number of LTA during restructuring 